\title{
Evidências de validade de uma escala para ansiedade em situações de mentira
}

\author{
Silvio José Lemos Vasconcellos ${ }^{1}$, Bruna Staevie dos Santos, \\ Lísia Quoos Morais, Raul Corrêa Ferraz, Pedro Osorio de Freitas, Jaíne Foletto Silveira \\ Universidade Federal de Santa Maria, Santa Maria-RS, Brasil
}

\section{RESUMO}

Mentir é um ato cotidiano de interação social. As pessoas mentem por todos os tipos de razões, e diferentes emoções são experimentadas nessas situações. Como parte integrante da cultura, a mentira tem recebido grande atenção de pesquisadores nas últimas décadas. No Brasil, esse novo campo de pesquisa tem atraído interesse nos últimos anos. Este estudo foi criado para construir e validar uma medida da ansiedade relacionada ao ato de mentir. Os 44 itens elaborados para representar o constructo foram aplicados a uma amostra de 452 indivíduos. Os dados foram submetidos a análise fatorial exploratória e dois fatores foram obtidos com valores próprios maiores de 1,0. Fator 1: compromissos e negócios (10 itens, $\alpha=0,81$ ); fator 2: relacionamentos (10 itens, $\alpha=0,82)$. O instrumento resultante deste estudo para medir esse tipo de ansiedade abre caminhos para possíveis futuras pesquisas nacionais.

Palavras-chave: mentira; ansiedade; validade.

\section{ABSTRACT - Validity evidence of a scale of anxiety in lying situations}

Lying is an everyday act of social interaction. People lie for all sorts of reasons, and different emotions are experimented in these situations. As an integral part of the culture, lying has received a great deal of attention by researchers in the last decades. In Brazil, this new field of research has attracted interest in recent years. This study was set up to construct and validate a measure of the anxiety related to the act of lying. The 44 items elaborated to represent the construct were applied to a sample of 452 individuals. Data were submitted to exploratory factorial analysis and two factors were taken with eigenvalues over 1,0. Factor 1 covered appointments and business ( 10 items, $\alpha=0.81$ ); factor 2 covered relationships (10 items, $\alpha=0.82$ ) The method for measuring this kind of anxiety resulting from this study opens possibilities for future national research.

Keywords: lying; anxiety; validity.

\section{RESUMEN - Evidencias de validez de una escala para ansiedad en situaciones de mentiras}

Mentir es un acto cotidiano de la interacción social. La gente miente por todo tipo de razones y emociones diferentes que se experimentan en estas situaciones. Como parte integral de la cultura, la mentira ha recibido gran atención por parte de los investigadores durante las últimas décadas. En Brasil, este nuevo campo de pesquisa ha despertado el interés en los últimos años. Este estudio se creó para construir y validar una medida de la ansiedad relacionada con el acto de mentir. Los 44 ítems elaborados para representar el constructo se aplicaron a una muestra de 452 personas. Se presentaron datos para un análisis exploratorio factorial y dos factores fueron obtenidos con valores propios mayores que 1,0. El factor 1 cubrió citas y negocios (10 ítems, $\alpha=0,81)$ y el factor 2 cubrió relacionamientos (10 ítems, $\alpha=0,82)$. El método resultante de eso estudio para medir este tipo de ansiedad abre posibilidades para futuras investigaciones nacionales.

Palabras clave: mentira; ansiedad; validad.

$\mathrm{Na}$ atualidade, o estudo sobre os comportamentos verbais e não verbais associados à mentira e à omissão de informações representa uma área de interesse crescente para a Psicologia, bem como para outras áreas afins. Profissionais que atuam no contexto forense constantemente deparam-se, por exemplo, com situações nas quais um melhor entendimento sobre a veracidade ou falsidade de um depoimento revela-se necessária. Ainda que esse conhecimento não seja, por si só, suficiente para subsidiar decisões judiciais, entende-se que pode melhor orientar as avaliações ou inquirições que ocorrem nesse mesmo contexto.

O desenvolvimento de novas pesquisas voltadas para as possibilidades e os limites no que se refere à detecção de mentira em situações interpessoais é, portanto, justificável e pode, em termos interdisciplinares, 
mostrar-se promissor quando devidamente embasado. A elaboração e validação de novos protocolos de entrevista, softwares que viabilizem o treinamento relacionado à detecção de incongruências verbais e não verbais, bem como escalas voltadas para avaliar os correlatos da mentira são empreendimentos pertinentes. De outro modo, observa-se que, no Brasil, essa instrumentalização ainda é incipiente para contribuir com as pesquisas na área, conforme evidencia a busca em portais científicos relacionada a instrumentos de ansiedade para situações específicas. No que se refere especificamente aos trabalhos sobre detecção e crenças relacionadas à mentira em nosso país, um trabalho de revisão da literatura corroborou o número ainda pequeno de estudos nesses campos (Matias, Leme, Bezerra, \& Torro-Alves, 2015). Já em outros países, constata-se que uma série de pesquisas se valem de instrumentos construídos para esses mesmos fins.

O Criteria-Based Content Analysis (CBCA) analisa, por exemplo, o conteúdo de um depoimento com base em 19 critérios. Trata-se de um protocolo para acompanhar a aplicação de dispositivos como o polígrafo, que, nesses termos, também considera a ansiedade envolvida no ato de mentir. É aceito em contexto forense em países europeus, como Holanda, Áustria e Suíça, e nos EUA (Köhnken, 2004; Ruby \& Brigham, 1997). A acurácia do CBCA é reportada de formas distintas, com valores entre 58\% (Granhag, Strömwall, \& Landström, 2006) e próximos a 90\% (Akehurst, Köhnken, \& Höfer, 2001).

O Control Question Test (CQT) é um protocolo constituído por cinco fases (Raskin \& Honts, 2002). A primeira, denominada fase pré-entrevista, visa a obtenção de informações sobre o sujeito e o crime, seguida do teste de estímulo. Nas demais fases, são realizas perguntascontrole e perguntas relevantes, que são seguidas de uma análise. A acurácia do CQT em estudos de laboratório é estimada em 77\% (Honts \& Quick, 1995) na porcentagem de acertos para classificar indivíduos culpados e de 63\% (Shakhar \& Furedy, 1990) a 84\% (Raskin \& Honts, 2002) para classificar indivíduos inocentes. De outro modo, constata-se que em tais situações de laboratório, o grau de ansiedade específica envolvendo o ato de mentir não tem sido controlado como forma de melhor testar os referidos protocolos.

Estudos de laboratório envolvendo o aumento da carga cognitiva têm sido realizados, obtendo dados sugestivos, porém, sem o controle da variável anteriormente aludida. A premissa principal dos trabalhos nessa área refere-se ao fato de que, sob certas condições, mentir exige esforços cognitivos maiores do que falar a verdade. A abordagem consiste em produzir situações que aumentem a carga cognitiva para o entrevistado. Sujeitos mentindo produzem mais pistas para a classificação se solicitados, por exemplo, a contar os acontecimentos na ordem inversa (Vrij, Mann, Fisher, Leal, Milne, \& Bull, 2008). Instruir suspeitos a manter contato visual durante a entrevista também produziu efeitos semelhantes (Doherty-Sneddon, Bruce, Bonner, Longbotham, \& Doyle, 2002; Walczyk, Griffith, Yates, Visconte, Simoneaux, \& Harris, 2012).

Nessa mesma perspectiva, Debey, Verschuere e Crombez (2012) evidenciaram um maior uso de recursos cognitivos ligados às funções executivas do cérebro, valendo-se de diferentes testagens neuropsicológicas. Tais achados foram também confirmados por Visu-Petra, Miclea e Visu-Petra (2012). No entanto, somente o último trabalho controlou o fator ansiedade, que se revelou interveniente nesse processo.

Em termos gerais, entende-se que a ansiedade está diretamente relacionada a uma maior ou menor intensidade no que se refere aos sinais comumente associados à mentira, mesmo em estudos de laboratório. Essa relação é evidenciada por intermédio do uso de dispositivos que mensuram sinais fisiológicos envolvidos no ato de mentir e que apresentam relação direta com a variável ansiedade. Conforme Vrij (2008), a Detecção Psicofisiológica da Mentira (PDD - Psychophysiological Deception Detection tests) recorre a medidas como atividade eletrodermal, pressão arterial, frequência cardíaca, frequência respiratória e atividade elétrica encefálica em aparatos comumente denominados como polígrafos. Para tanto, uma linha de base capaz de diferenciar tais reações em diferentes momentos do interrogatório se monstra imprescindível. Também é necessário ressaltar que existe um padrão individual envolvendo $\mathrm{o}$ ato de mentir ou mesmo associado a determinados quadros psicopatológicos. Alterações comportamentais bastante específicas têm sido verificadas em psicopatas quando comparados ao grupo controle em tais situações. (Klaver, Lee, \& Hart, 2007). Foi constatado nesse grupo, por exemplo, maior movimento com a cabeça e fala mais rápida em situações de mentira quando comparados a indivíduos sem o diagnóstico, sugerindo o uso de estratégias mais automatizadas para despistar o interlocutor (Klaver, Lee, \& Hart, 2007).

Diferenças de gênero no que se refere ao tipo de mentira e sentimentos em relação às mesmas também têm sido reportados na literatura. Homens tendem mais a proferir mentiras relacionadas a circunstâncias pessoais, enquanto mulheres estariam mais propensas a proferir mentira envolvendo outros indivíduos (Vrij, 2009). Em um experimento conduzido por DePaulo e Bell (1996), os participantes foram solicitados a escolher dois tipos de pintura conforme as suas preferências e duas outras pinturas que não despertavam o seu interesse diante de 19 alternativas. Posteriormente, foram conduzidos a um encontro com um estudante de arte que seria o suposto autor de algumas dessas obras, mas que, conforme foi relatado, não sabia sobre as escolhas dos envolvidos. De um modo geral, observou-se que as mulheres, ao mentirem sobre suas preferências estéticas, expuseram mais sentimentos positivos na tentativa 
de convencer seus interlocutores sobre as suas preferências. Nesse estudo, a ansiedade dos participantes em relação a situações de mentira não foi, no entanto, controlada com o objetivo de parear as subamostras formadas por homens e mulheres.

A literatura científica também sugere que diferenças de gênero que perfazem o tipo de mentira que se verifica em homens e mulheres podem se dar de forma ainda mais específica. Homens tendem mais à realização de mentiras capazes de denegrir um rival no que se refere a uma disputa amorosa (Vrij, 2009). Mulheres, de outro modo, estão mais propensas a mentir sobre aspectos físicos relacionados à própria aparência diante de um parceiro em potencial (Vrij, 2009). Nessa mesma perspectiva, Toma, Hancock e Elisson (2008) demonstraram que 81\% das pessoas mentem sobre o seu peso, a sua altura ou a sua idade em relacionamentos a distância, sendo que os homens tendem a mentir mais sobre a altura e mulheres a mentir mais sobre o peso.

Explicações para essas diferenças coadunam-se com a teoria da interferência estratégica proposta por Buss (1989). Para o autor, uma vez que, em termos evolutivos, homens e mulheres depararam-se com problemas adaptativos distintos, tendências sociais específicas podem perfazer cada uma dessas realidades. Nesse sentido, homens e mulheres reagem de forma distinta às estratégias de enganação dos parceiros e vivenciam níveis de ansiedade diferenciados quando empregam estratégias de enganação (Haselton, Buss, Oubaid, \& Angleitner, 2005). Conforme essa perspectiva, são essas mesmas reações emocionais específicas que podem tornar determinados comportamentos, incluindo determinados tipos de mentira, mais ou menos recorrentes no ambiente social (Buss, 1989).

Diante dessas considerações, constata-se que se mostra importante controlar a variável ansiedade em pesquisas delineadas com o objetivo de melhor compreender os correlatos fisiológicos ou comportamentais da mentira ou mesmo em estudos comparativos de gênero. Além disso, estudos que buscam comparar diferentes grupos no que se refere à capacidade de detectar a mentira também podem controlar essa variável como forma de padronizar as situações de entrevista produzidas para esses fins. Torna-se importante, nesses termos, quantificar tendências específicas atreladas à ansiedade em tais situações como uma possível forma de melhor investigar os chamados vazamentos emocionais e as suas especificidades, incluindo as expressões e microexpressões faciais destacadas nos trabalhos de Ekman (2003).

Com o objetivo de suprir essa demanda, a presente pesquisa objetivou construir e buscar evidências de validade de uma Escala de Ansiedade em Situações de Mentira (EASME). Esse instrumento foi originalmente composto por 44 itens descritivos de situações de mentira, diante das quais o avaliado não é solicitado a responder se cometeu ou não a referida mentira. Busca-se, de outro modo, que o participante apenas sinalize, de forma indistinta, em uma escala Likert que varia de 1 até 5 pontos, o grau de ansiedade que já experimentou ou supõe que iria experimentar em cada uma das situações descritas. Na sequência deste artigo, os autores descrevem o processo de elaboração e validação desse instrumento de avaliação psicológica que poderá subsidiar novos estudos nesse campo. Entende-se, desse modo, que a escala em questão poderá servir como uma medida-controle para diferentes pesquisas feitas sobre expressão e detecção da mentira, viabilizando novos parâmetros para tais investigações. Além disso, resultados futuros poderão ainda indicar mentiras consideradas, em termos sociais, mais ou menos toleráveis, permitindo estudos comparativos em nosso país.

\section{Método}

\section{Participantes}

Para a validação de construto, a versão com 44 itens foi aplicada em uma amostra de 452 estudantes universitários dos cursos de Direito, Ciências Econômicas, Ciências Sociais, Relações Internacionais, Filosofia, Sociologia, Serviço Social, Ciências Contábeis, Comunicação Social e Letras de uma universidade da região sul do Brasil, sendo 256 do sexo feminino e 196 do sexo masculino, com média de idade de 22,3 anos, com desvio padrão de 8,5. O número de participantes deste estudo foi calculado para a obtenção de soluções fatoriais estáveis. Para tanto, foi utilizado o critério "razão itens/sujeito" que indica, de forma aproximada, essa relação para tornar a análise adequada. Conforme Pasquali (1999) e Hair, Balck, Babin, Anderson e Tatham (2006), uma proporção mínima de cinco por um, referente ao tamanho da amostra e o número de itens constitutivos da escala, mostra-se necessária para um levantamento apropriado das características psicométricas que podem ser reveladas a partir da análise fatorial. Salienta-se, no entanto, que o número de sujeitos na amostra foi superior a dez vezes o número de itens que constituiu a versão preliminar da escala.

\section{Procedimentos}

A presente pesquisa foi submetida a um Comitê de Ética em Pesquisa, tendo recebido aprovação para a sua execução. A elaboração dos itens considerou aspectos culturais relacionados à mentira (Barnes, 1996), bem como um levantamento de algumas mentiras consideradas socialmente mais toleráveis ou mesmo recorrentes em determinadas situações interpessoais, conforme um grupo de 15 estudantes universitários. Com base nesses critérios, foram elaborados 44 itens descritivos de situações de mentira a exemplo de "Mentir sobre o seu currículo em uma entrevista de emprego" ou "Mentir sobre o preço de um produto que você deseja revender para um estranho". Esses itens foram ajustados em termos 
semânticos e sintáticos a partir de uma validação de conteúdo realizada com três profissionais de Psicologia, sendo os mesmos pesquisadores voltados para o tema da mentira e dos comportamentos antissociais. Um total de oito itens sofreu ajustes nessa etapa, considerando que foram classificados como inadequados pelos avaliadores. O restante, perfazendo um total de 36 itens, foi classificado como adequado para avaliar o constructo. A partir das modificações realizadas, a versão com 44 itens foi considerada satisfatória e clara pelos 3 avaliadores em uma segunda avaliação, tendo por base ainda o grau de escolaridade da amostra pesquisada.

Nessa versão, não foram inseridos itens que levassem o avaliado a presumir, por exemplo, a ansiedade que ele sentiria ao omitir um crime contra a vida. De outro modo, alguns dos itens escolhidos ainda estavam relacionados a delitos menos graves, comumente concebidos, em termos jurisprudenciais, como infrações de menor potencial ofensivo. A não inclusão de itens descrevendo situações supostamente mais impactantes em termos emocionais objetivou minimizar qualquer tipo de rejeição ao preenchimento da escala na sua totalidade.

Para o cabeçalho desse instrumento, as instruções foram igualmente discutidas com um grupo de 15 estudantes universitários quanto à clareza e objetividade das mesmas. Na versão adotada com base nessa etapa de elaboração, consta, por exemplo, a observação de que não se trata de um teste com respostas corretas ou incorretas, além de destacar que o teste não objetiva avaliar se o indivíduo mente com frequência ou se já cometeu determinados tipos de mentira, mas tão somente quantificar a ansiedade que o participante considera que experimentaria diante do medo de ser desmascarado na situação descrita. O uso da palavra medo, nesse caso, foi considerado, pelos estudantes consultados, mais esclarecedor e capaz de ratificar que a escala mensura níveis presumidos de ansiedade, sem buscar dados confirmatórios quanto ao fato de tais situações terem ou não ocorrido com os participantes. Para a obtenção dos escores, foi utilizada uma escala Likert de cinco pontos com variações de nenhum grau de ansiedade até grau extremo de ansiedade em cada uma das situações descritas.

\section{Resultados}

Para a verificação da dimensionalidade da EASME, foram extraídas soluções fatoriais com dois e três fatores. Como a maior parte dos itens criados supostamente representava o mesmo construto (a ansiedade em situações de mentira), considerou-se mais adequada a utilização da rotação direct oblimin, objetivando a extração de fatores correlacionados, bem como o método análise dos componentes principais (ACP). O Valor KMO $(\mathrm{KMO}=0,93)$ indicou a adequação da amostra para a análise proposta. O teste de Bartlett à esfericidade apresentou um valor de $\chi^{2}=8625,449 ; p<0,001$; sugerindo a aludida adequação.

Uma solução de dois fatores foi considerada adequada, uma vez que preservou um número razoável de itens em cada fator, permitindo ainda a exclusão de itens com carga fatorial inferior a um valor de 0,30 no fator obtido. Esse valor é considerado aceitável para critério de exclusão (Kline, 1994). Realizou-se ainda a exclusão de itens com carga fatorial superior a 0,30 em mais de 1 dos fatores propostos após a avaliação da não adequação teórica desses itens em nenhum dos fatores resultantes. Esses critérios, conjuntamente, explicam a opção por excluir um número elevado de itens. Destaca-se que o modelo de dois fatores decorrente revelou ainda uma ótima consistência interna para todos os fatores obtidos, sendo que o mesmo não ocorreu para o modelo de três fatores, sendo capaz de abarcar itens pouco semelhantes entre si no que se refere ao terceiro fator. A análise também considerou o método scree plot, sugerindo a possibilidade de uma solução de até três fatores para a EASME, conforme ilustra a Figura 1. Salienta-se que, conforme destaca Damásio (2012), o método scree plot pode oferecer interpretações ambíguas, ainda que possa auxiliar na proposição dos fatores dos instrumentos de testagem. Os autores do presente trabalho consideraram, no entanto, a solução de dois fatores mais adequada e relativamente compatível com o citado método, servindo o mesmo, nesse caso, como um elemento adicional para que a referida solução de dois fatores fosse aceita, considerando também os demais dados decorrentes da análise fatorial destacados neste trabalho.

O primeiro desses fatores foi denominado "compromissos e negócios", sendo capaz de abarcar itens mais voltados para a mentira em situações de transação comercial, bem como itens envolvendo a mentira diante de compromissos assumidos em termos acadêmicos ou profissionais. Já o segundo fator foi denominado "relacionamentos", abarcando itens voltados para as relações interpessoais estabelecidas de forma recente ou mais antigas. Salienta-se que o primeiro fator se mostrou capaz de explicar 31,79\% da variância total e o segundo fator $5,70 \%$. A Tabela 1 exemplifica alguns itens que integram a versão final da escala, os fatores a que pertencem, a carga fatorial de cada item e a consistência interna de cada fator.

A Tabela 2 mostra a estatística descritiva para os dois fatores e para a escala na íntegra, além de explicitar as diferenças entre homens e mulheres na amostra investigada, objetivando testar a Teoria da Interferência Estratégica anteriormente aludida. Houve diferenças estatisticamente significativas na comparação dos dois grupos no que se refere ao primeiro fator $(t=-3,58$; $p<0,05 ; \mathrm{GL}=440)$, ao segundo fator $(t=-3,99 ; p<0,05$; $\mathrm{GL}=440)$, e ao resultado total da escala $(t=-3,99$; $p<0,05 ; \mathrm{GL}=440)$. Não houve correlação estatisticamente significativa entre os dois fatores e os escores total da escala com a idade dos avaliados. 


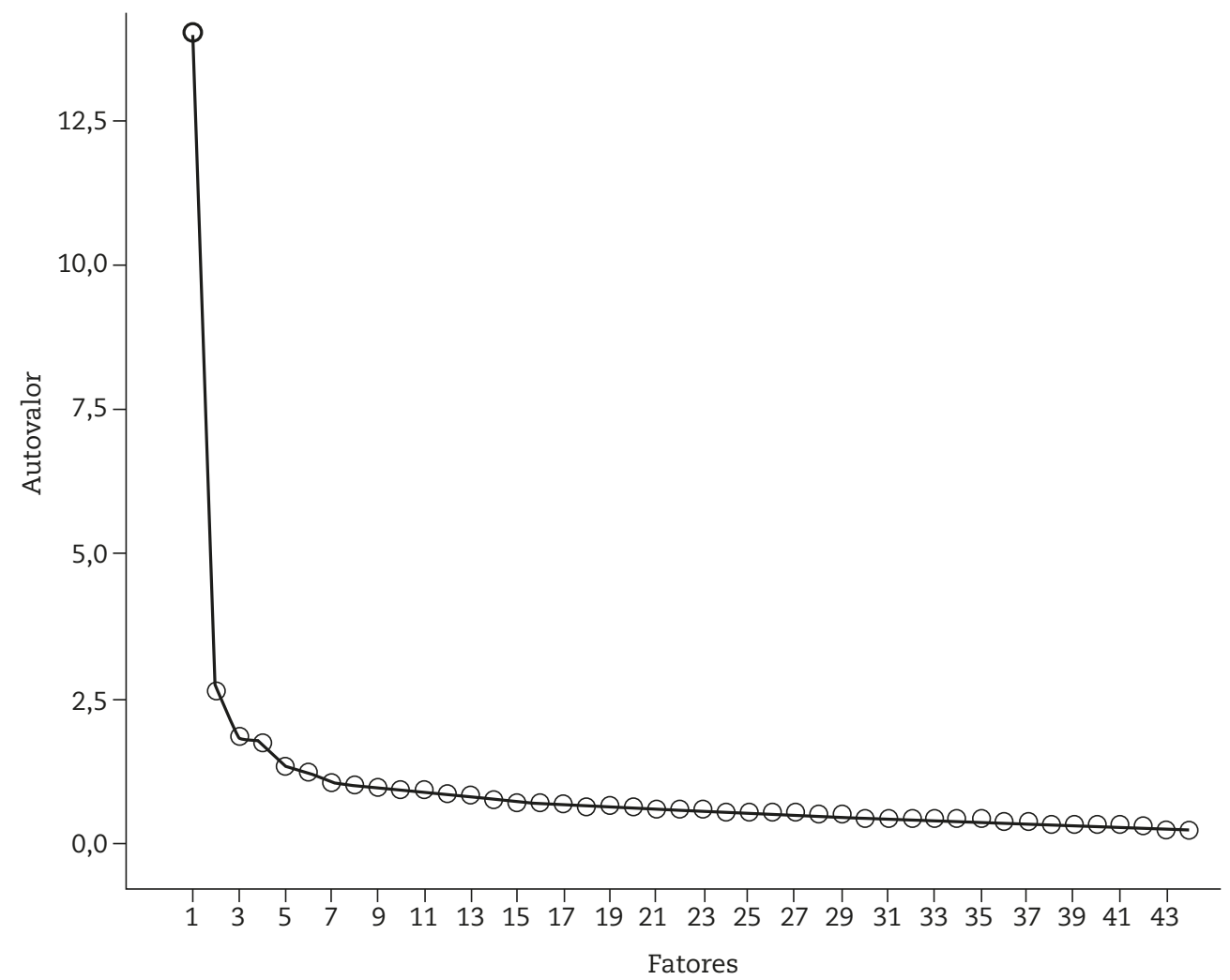

Figura 1. Gráfico de sedimentação da Escala de Ansiedade em Situações de Mentira (EASME)

Tabela 1

Exemplo de itens, fatores e carga fatorial da Escala de Ansiedade em Situações de Mentira

\begin{tabular}{|c|c|c|}
\hline Fatores & Itens & Carga fatorial \\
\hline \multirow{6}{*}{$\begin{array}{l}\text { Compromissos } \\
\text { e negócios } \\
\mu=0,81\end{array}$} & $\begin{array}{l}\text { Mentir perante um professor/superior, afirmando ser o único autor de um } \\
\text { trabalho copiado da internet }\end{array}$ & 0,45 \\
\hline & $\begin{array}{l}\text { Mentir sobre gastos médicos que nunca ocorreram em uma situação de } \\
\text { declaração do imposto de renda }\end{array}$ & 0,69 \\
\hline & $\begin{array}{l}\text { Mentir para uma seguradora sobre o tipo de acidente no qual você } \\
\text { envolveu-se para poder ser ressarcido }\end{array}$ & 0,54 \\
\hline & $\begin{array}{l}\text { Mentir sobre a sua renda familiar em uma loja na qual você está fazendo } \\
\text { um novo cadastro }\end{array}$ & 0,74 \\
\hline & $\begin{array}{l}\text { Mentir após ter quebrado um objeto em uma loja, afirmando que o mesmo } \\
\text { já estava quebrado }\end{array}$ & 0,63 \\
\hline & $\begin{array}{l}\text { Mentir que você não sabia que uma determinada nota era falsa, ao tentar } \\
\text { repassá-la para um comerciante }\end{array}$ & 0,38 \\
\hline \multirow{6}{*}{$\begin{array}{l}\text { Relacionamentos } \\
\mu=0,82\end{array}$} & $\begin{array}{l}\text { Mentir para um(a) novo(a) amigo(a) que o recebeu para um jantar que está } \\
\text { adorando a comida }\end{array}$ & 0,48 \\
\hline & $\begin{array}{l}\text { Mentir sobre as suas preferências estéticas (gostos) para não constranger } \\
\text { um artista que você acabou de conhecer }\end{array}$ & 0,63 \\
\hline & $\begin{array}{l}\text { Mentir que um trabalho recente de sua autoria recebeu uma premiação } \\
\text { profissional ou acadêmica para impressionar os colegas }\end{array}$ & 0,31 \\
\hline & $\begin{array}{l}\text { Mentir sobre a sua idade em uma conversa informal com pessoas para as } \\
\text { quais você foi recentemente apresentado }\end{array}$ & 0,40 \\
\hline & $\begin{array}{l}\text { Mentir que você não está mais magoado com alguém citado em uma } \\
\text { conversa, como forma de evitar que outras pessoas ao seu redor pensem } \\
\text { que você é alguém que guarda rancor }\end{array}$ & 0,52 \\
\hline & $\begin{array}{l}\text { Mentir sobre os seus filmes favoritos, como forma de impressionar uma } \\
\text { pessoa que você acabou de conhecer }\end{array}$ & 0,47 \\
\hline
\end{tabular}


Tabela 2

Médias e desvio padrão das subescalas e escala geral por sexo

\begin{tabular}{lcccccc}
\hline & \multicolumn{3}{c}{ Homens } & \multicolumn{3}{c}{ Mulheres } \\
\cline { 2 - 6 } & F1 & F2 & EASME & F1 & F2 & EASME \\
\hline Média & 30,46 & 28,99 & 59,45 & 33,02 & 31,67 & 64,7 \\
Desvio padrão & 8,13 & 7,54 & 14,84 & 6,88 & 6,56 & 12,74 \\
\hline
\end{tabular}

Nota: EASME=Escala de Ansiedade em Situações de Mentira.

\section{Discussão}

Um dos principais desafios referentes à elaboração da EASME relacionou-se com o pressuposto de que pessoas que mentem de forma mais frequente também podem mentir em um instrumento de autorrelato, ainda que o mesmo não tenha sido construído para fins de avaliação no contexto forense. Essa propensão foi parcialmente contornada pelo fato de que o instrumento elaborado propiciou uma quantificação específica no que se refere a um nível de ansiedade presumida, que por sua vez, prescinde de experiências anteriores em situações iguais ou semelhantes. Uma vez que os itens são descritivos de situações facilmente identificáveis e que não necessitam ser pormenorizadas, acredita-se que a escala proposta mostrou-se adequada quanto aos objetivos destacados, conforme sugerido na etapa de elaboração do instrumento e validação de constructo descritas neste manuscrito. A EASME apresentou uma solução de dois fatores satisfatória, revelando bons níveis de consistência interna em cada um dos fatores constitutivos do instrumento. Também podese ressaltar o tamanho adequado da versão resultante. Uma vez que o instrumento não está voltado para dimensões ou facetas diversificadas da personalidade, ou mesmo para sintomas que caracterizam síndromes complexas, mas tão somente para medir uma manifestação bastante específica da ansiedade, entende-se que a versão final poderá mostrar-se funcional e útil para pesquisas futuras.

Destaca-se o fato de que a EASME é um instrumento que foi elaborado para subsidiar pesquisas futuras no campo da detecção da mentira. Considera-se cabível que os estudos voltados, por exemplo, para comparar o desempenho de diferentes categorias profissionais, possam avaliar se o mesmo é afetado pelo nível de ansiedade dos sujeitos escolhidos como protagonistas das entrevistas. Em muitos estudos desse tipo, criam-se situações artificiais, muitas delas envolvendo estudantes universitários, que são solicitados a proferir verdades ou mentiras sobre circunstâncias específicas, preferências ou pontos de vista sobre temas polêmicos (Vrij, 2009). O controle da variável ansiedade relacionada à mentira por si só não acarretaria uma maior validade ecológica nesses trabalhos, mas pode, por certo, refinar os recursos usados para avaliar tais capacidades, incluindo a aplicação de alguns protocolos anteriormente destacados (Raskin \& Honts, 2002).

Outra possibilidade para a utilização da EASME diz respeito ao campo da Psicopatologia. Faz-se necessário melhor investigar os correlatos de alguns transtornos mentais em termos de aspectos bastante específicos da cognição social, a exemplo da psicopatia. Particularidades desse tipo podem gerar avanços quanto ao entendimento de transtornos do espectro autista, uma melhor compreensão do transtorno de ansiedade social, bem como das manifestações específicas de determinados transtornos de personalidade, além de outros. No caso da psicopatia, um dos instrumentos mais difundidos para o diagnóstico, a denominada escala Hare, avalia a mentira patológica que consta como um dos itens do referido checklist. Porém, exceto por medidas exclusivamente fisiológicas, essa manifestação específica da psicopatia tem sido pouco avaliada no que se refere aos seus correlatos (Klaver, Lee, \& Hart, 2007). Dessa forma, um instrumento de ansiedade em situações de mentira pode gerar dados adicionais para complementar essa investigação, uma vez que o próprio transtorno pode ser melhor compreendido em termos dimensionais e não exatamente como uma categoria fixa.

$O$ instrumento cuja validação encontra-se descrita neste trabalho pode ainda gerar dados que permitam comparações culturais em amostras mais amplas. Deve-se ressaltar que os dados ainda não são suficientes para um estudo de normatização, considerando o próprio fato de que estão restritos a uma única região do Brasil. Entretanto, trabalhos futuros poderão ampliar a amostra e viabilizar a referida normatização da escala. Novos estudos desse tipo poderão, por exemplo, revelar diferenças culturais sutis considerando a vastidão do território brasileiro.

São igualmente destacáveis as diferenças obtidas quanto ao nível de ansiedade em situações de mentiras envolvendo homens e mulheres. Embora a literatura científica acabe por sugerir distinções quanto à própria motivação para mentir envolvendo homens e mulheres, essas mesmas distinções não se estendem para o nível de ansiedade vivenciado quanto ao tipo de mentira ou os vazamentos emocionais daí decorrentes (Vrij, 2009). De outro modo, observou-se neste trabalho uma diferença estatisticamente significativa, indicando maior 
ansiedade para mulheres no que se refere às mentiras envolvendo relacionamentos, bem como em situações laborais ou de negociação. Esses dados sugerem a pertinência do entendimento relativo a possíveis interferências estratégicas que podem, de forma diferenciada e adaptativa, ter predisposto homens e mulheres a determinados comportamentos no ambiente social (Buss, 1989; Haselton et al., 2005). Uma hipótese que se coloca para pesquisas futuras é que tais diferenças podem ser averiguadas em um instrumento de autorrelato dimensionando diferenças mais sutis, mas que não chegam a afetar a frequência dessas mesmas mentiras. Em outras palavras, embora o nível de ansiedade das mulheres seja superior nos dois fatores, existem diferenças de gênero que tornam mais prováveis determinados tipos de mentira para homens e determinados tipos de mentira para mulheres, conforme destaca Vrij (2009).

Estudos posteriores podem investigar de forma mais pormenorizada a relação entre ansiedade geral e a ansiedade envolvendo o ato de mentir, além da relação desse último constructo com a ansiedade social. Tais dados poderão também contribuir para uma maior elucidação quanto aos diferentes graus de ansiedade relacionada à mentira em homens e mulheres. Sabe-se, por exemplo, que mulheres apresentam maior nível de ansiedade geral, bem como uma maior prevalência de transtorno de ansiedade generalizada (Wittchen, 2002). Por outro lado, a ansiedade relacionada ao ato de mentir não é constitutiva de uma patologia específica e pode ser mais bem explicada a partir dos valores que um indivíduo adota para conduzir a própria vida, bem como por outras variáveis a exemplo da empatia (Barnes, 1996).
Como uma das limitações do presente estudo, destaca-se a homogeneidade da amostra, composta na sua totalidade de estudantes universitários. Em amostras mais diversificadas, poder-se-ia observar, por exemplo, diferenças relacionadas à atuação profissional dos participantes, bem como o próprio tempo de atuação como variáveis intervenientes. Além disso, o presente trabalho limitou-se à utilização da análise fatorial exploratória no campo da Psicometria. Outras análises poderão complementar o presente trabalho.

Em termos mais conclusivos, a versão inicial da EASME obtida após a validação de conteúdo realizada foi considerada de fácil compreensão, sendo a coleta de dados considerada, conforme os juízes consultados, exequível com uma população com ensino médio completo. A partir disso, optou-se por realizar o estudo de validação da escala com uma amostra de estudantes universitários de diferentes cursos, principalmente da área de Ciências Humanas.

Os dados obtidos sugerem que a EASME é um instrumento válido para pesquisas futuras no contexto brasileiro. Poderá, portanto, fundamentar estudos que ainda se mostram incipientes no Brasil, mas que despertam cada vez mais o interesse dos profissionais e estudantes de Psicologia em nosso país. Considera-se, desse modo, que a versão final pode ser de fácil aplicação, podendo fornecer índices que poderão viabilizar estudos comparativos a partir de um estudo de normatização posterior. Alguns estudos correlacionais com outros instrumentos poderão ainda evidenciar que a ansiedade em situações de mentira apresenta-se como um constructo singular que necessita ser controlado, principalmente em estudos voltados para a detecção da mentira.

\section{Referências}

Akehurst L., Köhnken G., \& Höfer E. (2001). Content credibility of accounts derived from live and video presentations. Legal and Criminological Psychology, 6(1),65-83. doi: 10.1348/135532501168208

Barnes, J. A. (1996). Um monte de mentiras: para uma sociologia da mentira. Campinas: Papirus.

Buss, D. M. (1989). Conflict between de sexes: Strategic interferences and the evocation of anger and upset. Journal of Personality and Social Psychology, 56(5), 735-747.

Damásio, B. F. (2012). Uso da análise fatorial exploratória em Psicologia. Avaliação Psicológica, 11(2), 213-228.

Debey, E., Verschuere, B., \& Crombez, G. (2012). Lying and executive control: An experimental investigation using ego depletion and goal neglect. Acta Psychologica, 140(2), 133-141. doi: 10.1016/j.actpsy.2012.03.004

DePaulo, B. M., \& Bell, K. L. (1996). Truth and investment: Lies are told to those who care. Journal of Personality and Social Psychology, 71(4), 703-716. doi: 10.1037/0022-3514.71.4.703

Doherty-Sneddon, G., Bruce, V., Bonner, L., Longbotham, S., \& Doyle, C. (2002). Development of gaze aversion as disengagement of visual information. Developmental Psychology, 38(3), 438-445. doi: 10.1037/0012-1649.38.3.438

Ekman, P. (2003). Emotions revealed: Recognizing faces and feelings to improve communication and emotional life. New York: Times Book.

Granhag, P. A., Strömwall, L. A., \& Landström, S. (2006). Children recalling an event repeatedly: Effects on RM and CBCA scores. Legal and Criminological Psychology, 11(1), 81-98. doi: 10.1348/135532505X49620

Hair, Jr., J. F., Black, W. C., Babin, B. J., Anderson, R. E., \& Tatham, R. L. (2006). Multivariate data analysis. 6a edição. Upper Saddle River, NJ: Pearson Prentice Hall.

Haselton M. G., Buss, D. M., Oubaid, V., \& Angleitner, A. (2005). Sex, lies and strategic interferences: The psychology of deception between the sexes. Personality \& Social Psychology Bulletin, 31(1), 3-23. doi: 101177/0146167204271303 
Honts, C. R., \& Quick, B. D. (1995). The polygraph in 1995: Progress in science and the law. North Dakota Law Review, 7(1), $1987-1020$.

Kline, P. (1994). An easy guide to factor analysis. New York: Routledge.

Klaver, J. R., Lee, Z., \& Hart, S. D. (2007). Psychopathy and nonverbal indicators of deception in offenders. Law and Human Behavior, 31(4), 337-351. doi: 10.1007/s10979-006-9063-7

Köhnken, G. (2004). Statement valifity analysis and the 'detection of the truth'. In P. A. Granhag \& L. A. Strömwall (Eds.). The detection of deception in forensic contexts (pp. 41-63). Cambridge, UK: Cambridge University Press.

Matias, D. W. S., Leme, J. L., Bezerra, C. W. A. G., \& Torro-Alves, N. (2015). Mentira: aspectos sociais e neurobiológicos. Psicologia: Teoria e Pesquisa, 31(3), 397-401. doi: 10590/0102-37722015032213397401

Pasquali, L. (1999). Testes referentes a construto: teoria e modelo de construção. In L. Pasquali (Org.). Instrumentos psicológicos: Manual prático de elaboração (pp. 37-71). Brasília, DF: Laboratório de Pesquisa em Avaliação e Medida - LabPAM.

Raskin, D. C., \& Honts, C. R. (2002). The comparison question test. In M. Kleiner (Ed.). Handbook of polygraph testing (pp.1-48). London, UK: Academic Press.

Ruby, C. L., \& Brigham, J. C. (1997). The usefulness of the criteria-based content analysis technique in distinguishing between truthful and fabricated allegations: A critical review. C. L. Psychology. Public Policy, and Law, 3(4), 705-737. doi: 10.1037/1076-8971.3.4.705

Shakhar, G. B., \& Furedy, J. J. (1990). Theories and applications in the detection of deception: A psychophysiological and international perspective. New York, NY: Springer - Verlag Publishing.

Toma, C. L., Hancock, J. T., \& Elisson, N. B. (2008). Separating fact from fiction: An examination of deceptive self-presentation in online dating profiles. Personality E Social Psychology Bulletin, 34(8), 1023-1036.

Visu-Petra, G., Miclea, M., \& Visu-Petra, L. (2012). Reaction time-based detection of concealed information in relation to individual differences in executive functioning. Applied Cognitive Psychology, 26(3), 342-351. doi: 10.1002/acp.1827

Vrij, A. (2009). Detecting lies and deceit: Pitfalls and opportunities. 2nd edition. England: Jonh Wiley \& Sons, Ltd.

Vrij, A., Mann, S. A., Fisher, R. P., Leal, S., Milne, R., \& Bull, R. (2008). Increasing cognitive load to facilitate lie detection: The benefit of recalling an event in reverse order. Law and Human Behavior, 32(3), 253-265. doi: 10.1007/s10979-007-9103-y

Walczyk, J. J., Griffith, D. A., Yates, R., Visconte, S. R., Simoneaux, B., \& Harris, L. L. (2012). Lie detection by inducing cognitive load: Eye movements and other cues to the false answers of "witnesses" to crimes. Criminal Justice and Behavior, 39(7), 887-909. doi: $10.1177 / 0093854812437014$

Wittchen, H. U. (2002). Generalized anxiety disorder: Prevalence, burden, and cost to society. Depression and Anxiety, 16(4),162-71. doi: $10.1002 /$ da. 10065

\section{Sobre os autores}

Silvio José Lemos Vasconcellos é professor dos cursos de graduação e pós-graduação em Psicologia da Universidade Federal de Santa Maria e coordenador do grupo Pesquisa e Avaliação de Alterações da Cognição Social - PAACS.

Bruna Staevie dos Santos é graduanda (2012-2016) em Psicologia pela Universidade Federal de Santa Maria, integrante do Grupo PAACS.

Lísia Quoos Morais é graduanda (2013-2017) em Psicologia pela Universidade Federal de Santa Maria, integrante do Grupo PAACS.

Raul Corrêa Ferraz é graduando em Psicologia (2013-2017) pela Universidade Federal de Santa Maria, integrante do grupo PAACS e atualmente bolsista de iniciação científica FIPE Sênior.

Pedro Osorio de Freitas é graduando (2013-2017) em Psicologia pela Universidade Federal de Santa Maria, integrante do grupo PAACS e bolsista PIBIC CNPq de iniciação científica.

Jaíne Foletto Silveira é psicóloga (2010-2015) pela Universidade Federal de Santa Maria, atualmente faz mestrado em Psicologia (UFSM) com bolsa CNPQ e integrante do grupo PAACS. 